\title{
miR-608 inhibits the migration and invasion of glioma stem cells by targeting macrophage migration inhibitory factor
}

\author{
ZHENHUA WANG $^{1}$, YIXUE XUE $^{2}$, PING WANG $^{2}$, JIAQI ZHU $^{1}$ and JUN MA ${ }^{2}$ \\ Departments of ${ }^{1}$ Physiology and ${ }^{2}$ Neurobiology, College of Basic Medicine, China Medical University, \\ Shenyang, Liaoning 110122, P.R. China
}

Received November 25, 2015; Accepted December 31, 2015

DOI: $10.3892 /$ or.2016.4652

\begin{abstract}
Glioma stem cells (GSCs) contribute to the malignant biological behavior of these tumors and have also been shown to be resistant to radiation and chemotherapy. Recently, a variety of microRNAs (miRNAs) has been found to present altered expression and to play an important oncogenic role or tumor-suppressive function in cancer stem cells (CSCs). microRNA-608 (miR-608) is one of the newly discovered microRNAs, and its biological functions remain unclear. Human macrophage migration inhibitory factor (MIF) is a well known oncogene associated with tumor recurrence and the poor prognosis of gliomas. In the present study, we found that miR-608 negatively regulated the gene expression of MIF at the post-transcriptional level and plays a tumor-suppressive role by targeting MIF in GSCs. We found that miR-608 expression was significantly downregulated and the expression levels of the MIF gene and protein showed an increase in the GSCs. miR-608 overexpression significantly attenuated the proliferation, migration and invasion, and induced the apoptosis of GSCs. The dual-luciferase reporter system revealed that the
\end{abstract}

Correspondence to: Professor Zhenhua Wang, Department of Physiology, College of Basic Medicine, China Medical University, 77 Puhe Road, Shenyang North New Area, Shenyang, Liaoning 110122, P.R. China

E-mail:wzhcmusl@163.com

Abbreviations: GSCs, glioma stem cells; miRNA, microRNA; MIF, macrophage migration inhibitory factor; GBM, glioblastoma; CSCs, cancer stem cells; PI3K, phosphatidylinositol 3-kinase; JNK, Jun N-terminal kinase; MAP, mitogen-activated protein; HEK, human embryonic kidney; DMEM, Dulbecco's modified Eagle's medium; FBS, fetal bovine serum; bFGF, basic fibroblast growth factor; EGF, epidermal growth factor; qRT-PCR, real-time quantitative polymerase chain reaction; UTR, untranslated region; MTT, methyl thiazolyl tetrazolium; DMSO, dimethyl sulfoxide; TBST, Tris-buffered saline-Tween-20; ECL, enhanced chemiluminescence; IDVs, integrated density values; SD, standard deviation; ERK, extracellular signal-regulated kinases

Key words: miR-608, glioma stem cells, macrophage migration inhibitory factor
3'UTR of MIF is a direct target of miR-608, and miR-608 negatively regulates the gene expression of MIF at the posttranscriptional level by targeting its 3'UTR. Furthermore, we demonstrated that miR-608 overexpression inhibited the malignant behavior of GSCs by downregulating MIF. Western blot results showed that the inhibition of MIF resulted in the inactivation of the PI3K/AKT and JNK pathways. These results demonstrate that miR-608 acts as a potential tumor suppressor and provide insight into new therapeutic targets for malignant glioma.

\section{Introduction}

Glioblastoma (GBM) is the most common and lethal primary brain tumor widely invading surrounding brain tissues. GBM patients almost always suffer from tumor recurrence and have a less optimistic median survival period even after they receive conventional treatments involving surgery, radiation, as well as adjuvant chemotherapy. Studies demonstrate that gliomas and other primary brain tumors contain cancer stem cells (CSCs) which have the ability to self-renew and give rise to differentiated progeny $(1,2)$. These multipotent stem cells participating in the development and progressive growth of tumors have also been shown to be resistant to radiation and chemotherapy $(3,4)$. It has been reported that glioma stem cells (GSCs) contribute to the malignant biological behaviors of gliomas such as infiltrating growth, therapeutic resistance and high recurrence rates. Many therapeutic strategies for GSCs have been developed for GBM and other brain tumors (5). GSCs are key drivers to promote tumor growth by several complicated molecular mechanism including genetics, epigenetics, metabolism as well as various extrinsic regulatory factors. Recently, many investigators have found that a variety of microRNAs (miRNAs) present altered expression and play an important oncogenic role or tumor-suppressive function in CSCs (6).

MicroRNAs (miRNAs) are a type of small, singlestranded endogenous RNA molecules with a length of $\sim 21$ to 25 nucleotides present in eukaryotic cells, which regulate target gene expression at the post-transcriptional level by degrading target mRNAs or inhibiting translation. Studies have shown that miRNAs, acting as tumor promoters or suppressors, may modulate tumor initiation, development and progressive growth in a wide variety of tumors. miR-608 is 
one of the newly discovered microRNAs, and its biological functions are not clearly understood. One study showed that miR-608 acts as a tumor-suppressive microRNA regulating malignancy in chordoma (7). In the present study, we found that miR-608 expression was significantly downregulated in glioblastoma tissues and GSCs using real-time PCR, and the overexpression of miR-608 attenuated the invasion of GSCs; no literature has reported this finding thus far. Therefore, we investigated the role and detailed mechanisms of miR-608 in glioma.

In the present study, our results of the bioinformatic analysis predicted that the 3 'UTR of human macrophage migration inhibitory factor (MIF) contained a region (nucleotides 73-80) with perfect complementarity to miR-608. Luciferase reporter assay revealed that miR-608 negatively regulated the gene expression of MIF at the post-transcriptional level by direct interaction with the MIF 3'UTR target sites. Furthermore, we verified that the expression of the MIF gene and protein was significantly increased in GSCs using real-time PCR and western blotting. Studies suggest that MIF has a prominent role in regulating cell survival, proliferation, differentiation, and angiogenesis as well as promoting the occurrence and development of tumors. It has been reported that elevated expression of MIF correlates with tumor recurrence and poor prognosis of patients with gliomas $(8,9)$, but the association between MIF and GSCs remains unknown. We hypothesized that low expression of miR-608 in GSCs may prohibit its suppressive effect on the potential oncogene MIF at the transcriptional level and the expression of the MIF gene is consequently upregulated.

It has been suggested that the phosphatidylinositol 3-kinase (PI3K)/Akt signaling pathway may facilitate the proliferation and invasion of GBM cells, and blockage of PI3K-mediated signaling has a potent anti-proliferative and anti-invasive effect on GBM (10). In addition, Jun N-terminal kinase (JNK) belongs to one of three major mitogen-activated protein (MAP) kinase pathways, and it is clear that activation of the JNK pathway is involved in the induction of cell apoptosis (11). However, investigators have uncovered that the JNK pathway has dual roles in the malignant biological behavior of cancers. Activation of JNK may favor the cell migration of bladder cancer cells. It has been recently reported that ERK and JNK inhibitors effectively inhibited the migration of glioma cells $(12,13)$. Therefore, the PI3K/AKT and JNK signaling pathways are critical in the therapeutic approach to gliomas. Moreover, it was reported that MIF may activate the JNK signaling pathway in T cells and fibroblasts (14). MIF induced the expression of thrombomodulin and intercellular adhesion molecule-1 via JNK and PI3K pathways in human monocytoid (THP-1) cells (15). We questioned whether MIF may regulate the biological behaviors of GSCs via the PI3K/AKT or JNK signaling pathways.

In the present study, we aimed to prove the direct interaction between the seed sequence of miR-608 and MIF 3'UTR target sites. Then, we establish GSC models with overexpression and knockdown of miR-608 to demonstrate the regulatory actions of miR-608 on the expression and function of the MIF gene and the biological behavior of GSCs. The results of the study demonstrated the molecular mechanisms of miR608-mediated regulation of the biological behavior of GSCs by downregulating the expression of the MIF gene. These findings will help us to reveal the pathogenesis of brain glioma and provide new therapeutic targets for malignant glioma.

\section{Materials and methods}

Clinical specimens. Glioblastoma specimens were obtained from the Department of Neurosurgery, Shengjing Hospital of China Medical University. Fresh human brain tissues obtained by surgery for malignant intracranial hypertension were used as control. Immediately after surgical resection, the specimens were frozen and preserved in liquid nitrogen. According to WHO 2007 classification, the glioma specimens were classified into four grades, and then they were divided into a low-grade glioma group (WHO I-II, $n=3$ ) and a high-grade glioma group (WHO III-IV, n=3). All procedures were approved by the Ethics Committee of the China Medical University. All the specimens were obtained with the consent of the patients before surgery and written informed consent was received from all participants.

Cell culture and GSC isolation. Human GBM cell lines (U87 and U251) and human embryonic kidney (HEK) 293T cells were purchased from Shanghai Institutes for Biological Sciences Cell Resource Center. The cells were cultured in high-glucose Dulbecco's modified Eagle's medium (DMEM) containing 10\% fetal bovine serum (FBS; Life Technologies Corporation, Paisley, UK). When the U87 and U251 cells reached the logarithmic growth phase, they were resuspended in serum-free DMEM/F12 supplemented with $20 \mathrm{ng} / \mathrm{ml}$ of basic fibroblast growth factor (bFGF), $20 \mathrm{ng} / \mathrm{ml}$ of epidermal growth factor (EGF) and B27 serum-free supplement (all from Life Technologies Corporation). All cells were incubated at $37^{\circ} \mathrm{C}$ in a $5 \% \mathrm{CO}_{2}$ humidified incubator. The isolation and identification of GSCs from the U87 and U251 cells were carried out as described previously $(16,17)$. The GSCs successfully isolated from U87 and U251 were used for the subsequent study.

Qualitative real-time PCR ( $q R T$-PCR). Total RNA was extracted from the non-GSCs and GSCs with TRIzol reagent (Life Technologies Corporation) according to the manufacturer's protocol. The expression level of miR-608 mRNA was determined using TaqMan MicroRNA Reverse Transcription kit and TaqMan Universal Master Mix II (Applied Biosystems Carlsbad, CA, USA). The level of MIF mRNA was detected using PrimeScript ${ }^{\circledR}$ RT Master Mix Perfect Real-Time and SYBR $^{\circledR}$ Premix Ex Taq ${ }^{\mathrm{TM}}$ (Takara, Dalian, Liaoning, China). The mRNA levels of miR-608 and MIF were calculated by the relative quantification $\left(2^{-\Delta \Delta C t}\right)$ method using U6 and GAPDH as endogenous controls.

Cell transfection. GSCs were transfected with miR-608 mimic, miR-608 inhibitor or their respective negative control (NC, non-targeting sequence) which were synthesized by Suzhou GenePharma Co., Ltd. Opti-MEM ${ }^{\circledast}$ I and Lipofectamine 2000 reagent (Life Technologies Corporation) were used for transfection. The cells were harvested after a 48-h transfection, and the levels of miR-608 overexpression and inhibition in the transfected cells were determined by qRT-PCR. For investigating the roles of miR-608 on GSCs, 
the transfected cells were divided into five groups as follows: control group, miR-608 mimic NC group, miR-608 mimic group, miR-608 inhibitor NC group, miR-608 inhibitor group. MIF overexpression plasmid was obtained by ligating the human full-length MIF gene with its 3'-untranslated region (UTR) into the pEX-2 plasmid vector (GenePharma, Suzhou, China). In order to knockdown MIF, short hairpin RNA was directed against the human MIF gene (GenePharma). The MIF overexpression(+) or inhibition(-) plasmid was steadily transfected into GSCs, and screened out using G418 after $48 \mathrm{~h}$ of transfection. The transfection efficiency was verified by qRT-PCR. For determining the role of PI3K/AKT and JNK pathways in this experiment, the co-transfected GSCs were divided into nine groups: control group, miR-608 mimic NC + $\mathrm{MIF}(+) \mathrm{NC}$ group, miR-608 mimic + MIF(+) group, miR-608 mimic NC + MIF(-) NC group, miR-608 mimic + MIF(-) group, miR-608 inhibitor $\mathrm{NC}+\mathrm{MIF}(+) \mathrm{NC}$ group, miR-608 inhibitor + MIF(+) group, miR-608 inhibitor NC + MIF(-) NC group, and miR-608 inhibitor + MIF(-) group.

MTT assay. GSCs in logarithmic growth phase were collected with serum-free DMEM/F12 medium, and seeded in 96-well plates. After transfection for $48 \mathrm{~h}, 20 \mu \mathrm{l}$ of methyl thiazolyl tetrazolium (MTT; Sigma, USA) was added to each well and the cells were incubated at $37^{\circ} \mathrm{C}$ for $4 \mathrm{~h}$. Then $150 \mu \mathrm{l}$ of dimethyl sulfoxide (DMSO; Genview, USA) was added to each well. The optical density value was measured at $490 \mathrm{~nm}$.

Flow cytometry. ApoScreen Annexin V FITC/PI Apoptosis detection kit (Baosai, Beijing) was used to detect cell apoptosis. After transfected for $48 \mathrm{~h}$, the cells were collected, washed, and then resuspended in $200 \mu \mathrm{l}$ of Annexin $\mathrm{V}$ binding buffer. The cells were incubated with $10 \mu \mathrm{l}$ of Annexin V FITC at $4^{\circ} \mathrm{C}$. After incubation for $15 \mathrm{~min}, 300 \mu \mathrm{l}$ of cold binding buffer and $10 \mu \mathrm{l}$ of propidium iodide (PI) solution were added. Cells were analyzed with flow cytometry (FACScan; BD Biosciences).

Cell migration and invasion assays. The 24-well chambers with a polycarbonic membrane $(8-\mu \mathrm{m}$ pore size) were used for the cell migration assay. The cells were added to the upper chamber, incubated for $24 \mathrm{~h}$ with $100 \mu \mathrm{l}$ serum-free medium at the density of $5 \times 10^{5}$ cells $/ \mathrm{ml}$, and $600 \mu \mathrm{l}$ of medium with $10 \%$ FBS was added to the lower chamber. After incubation for $24 \mathrm{~h}$ at $37^{\circ} \mathrm{C}$, the chambers were removed and the cells on the upper surface of the chambers were wiped off. Cells that had migrated to the lower surface of the membrane were fixed with methanol and then stained with $20 \%$ Giemsa. The stained cells were observed and counted in five randomly selected fields using a microscope. For the cell invasion assay, the procedure was similar to the migration assay, but the upper chamber was precoated with $500 \mathrm{ng} / \mu \mathrm{l}$ Matrigel solution (BD Biosciences).

Luciferase assay. A Dual-luciferase reporter assay was performed to determine the effect of miR-608 on MIF 3'UTR and the functional binding sites in the MIF 3'UTR. MIF-3'UTR-Wt and MIF-3'UTR-Mut (GenePharma) were produced by subcloning the full-length 3'UTR fragments of the MIF gene and its mutant into the miR-608 binding sites into a pmirGlo Dual-Luciferase miRNA target expression vector
(Promega, Madison, WI, USA). HEK 293T cells were seeded in 96-well plates. After incubation for $24 \mathrm{~h}$ at $37^{\circ} \mathrm{C}$, the cells were co-transfected with MIF-3'UTR-Wt (MIF-3'UTR-Mut) and miR-608 (miR-608 NC). HEK 293T cells were divided into five groups as follows: empty vector group, miR-608 $\mathrm{NC}+$ MIF-3'UTR-Wt group, miR-608 + MIF-3'UTR-Wt group, miR-608 NC + MIF-3'UTR-Mut group, miR-608 + MIF-3'UTR-Mut group. After transfection for $48 \mathrm{~h}$, luciferase assays were performed using the Dual-Luciferase Reporter Assay system (Promega) and the firefly luciferase activity was normalized to Renilla luciferase activity.

Western blot assessment. Western blot analysis was used to detect the protein expression of MIF, PI3K, p-PI3K, AKT, p-AKT, JNK, and p-JNK in the GSCs. Total protein was extracted using RIPA buffer supplemented with protease inhibitors and phosphatase inhibitor. The protein concentration was determined using the BCA protein assay kit. Equal amounts of proteins were separated on 10 to $15 \%$ sodium dodecyl sulphate-polyacrylamide gels. The separated proteins were electrophoretically transferred to nitrocellulose, and then the membranes were blocked with 5\% nonfat milk in Tris-buffered saline-Tween-20 (TBST) overnight. The membranes were then incubated with the primary antibody for $2 \mathrm{~h}$, respectively as follows: MIF (rabbit, polyclonal, 1:500; BoAoSen, Beijing, China); JNK, p-JNK, PI3K, p-PI3K, AKT, p-AKT (rabbit, monoclonal, 1:1,000; Cell Signaling Technology, Danvers, MA, USA). After the protein was incubated with the secondary antibody conjugated with horseradish peroxidase for $2 \mathrm{~h}$ at room temperature, the immunoreactive bands were visualized by enhanced chemiluminescence (ECL kit; Santa Cruz Biotechnology, USA) and scanned with Chemi Imager 5500 V2.03 software. The relative integrated density values (IDVs) were calculated using a computerized image analysis system (Fluor Chen 2.0) and normalized with that of GAPDH.

Statistical analysis. All data are presented as the means \pm standard deviation (SD). Differences among multiple groups were analyzed using one-way ANOVA. Two sample t-tests were applied to test differences between groups. Statistical significance was assumed at a p-value $<0.05$.

\section{Results}

Downregulation of miR-608 expression is detected in glioblastoma tissues. The expression levels of miR-608 mRNA were analyzed in low-grade and high-grade glioma tissues by qRT-PCR. As shown in Fig. 1, miR-608 mRNA expression was significantly downregulated in glioblastoma tissues compared with the average expression levels in the control brain tissues $(\mathrm{P}<0.05)$. Moreover, the expression levels declined with the increasing pathological grades of the glioblastoma samples.

Low expression of miR-608 and high expression of MIF are verified in GSCs. The endogenous expression levels of miR-608 and MIF were evaluated in GSCs-U87 and GSCs-U251 using real-time PCR analysis. As shown in Fig. 2A and B, both miR-608 and MIF were expressed in malignant cells from the two types of glioma. The expression levels of miR-608 were significantly lower in the GSCs-U87 and GSCs-U251 


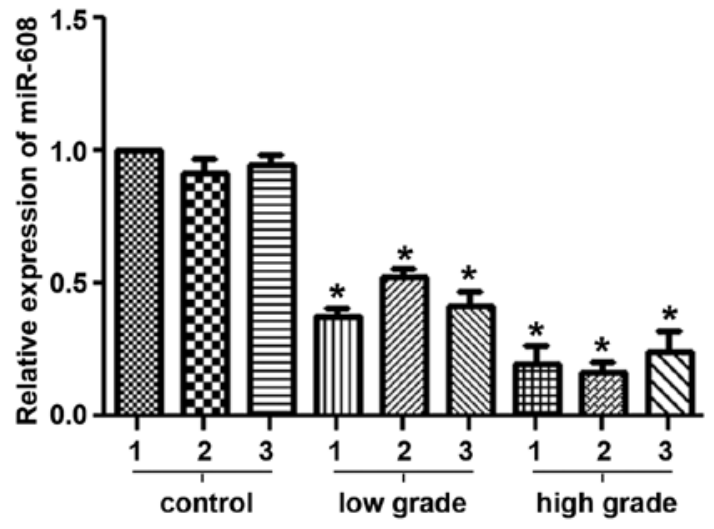

Figure 1. miR-608 is downregulated in human glioblastoma tissues. The expression level of miR-18a was assessed in control brain tissues, low-grade and high-grade glioma tissues by qRT-PCR. U6 was used as an internal control. Data are expressed as the mean $\pm \mathrm{SD}$ ( $\mathrm{n}=3$, each group). $\mathrm{P}<0.05$ vs. the control group.

than levels in non-GSCs-U87 and non-GSCs-U251 $(\mathrm{P}<0.05)$. By contrast, the expression level of the MIF gene was significantly increased in the GSCs $(\mathrm{P}<0.05)$. Western blot analysis revealed that the expression level of MIF protein also showed an increase in the GSCs-U87 and GSCs-U251 compared with the level in the non-GSCs $(\mathrm{P}<0.05$, Fig. 2C).

miR-608 overexpression inhibits the proliferation, migration and invasion, and induces the apoptosis of the GSCs. We explored the role of miR-608 in the malignant biological behaviors of human GSCs isolated from U87 and U251 cell lines via diversified functional analysis methods. MTT assays showed that the cell proliferation ability of the GSCs-U87 and GSCs-U251 transfected with the miR-608 mimic was significantly decreased compared with that noted in the miR-608 mimic NC group $(\mathrm{P}<0.05)$. In contrast, miR-608 inhibition increased the proliferation of the GSCs compared with that noted in the miR-608 inhibitor $\mathrm{NC}$ group ( $\mathrm{P}<0.05$, Fig. 3A).

As shown in Fig. 3B, the overexpression of miR-608 induced a prominent increased apoptosis in the GSCs, and the cells transfected with the miR-608 inhibitor showed a decreased apoptosis ability compared with that noted in the miR-608 inhibitor NC group $(\mathrm{P}<0.05)$. Furthermore, miR-608 to a large extent altered the migration and invasion abilities of the GSCs. The results of the cell migration and invasion assays revealed that miR-608 overexpression significantly attenuated the migration and invasion of the GSCs-U87 or GSCs-U251 compared with these abilities noted in the miR-608 mimic NC group, thus the migration and invasion abilities were strengthened in the GSCs-U87 and GSCs-U251 cells transfected with the miR-608 inhibitor $(\mathrm{P}<0.05$, Fig. 3C). All these results indicate that miR-608 is a type of miRNA that inhibits the malignant progression of GSCs.

Overexpression of miR-608 inhibits MIF expression by targeting its 3'UTR. The bioinformatic databases (TargetScan, PicTar, RNAhybrid) predicted that MIF may be one of the potential mRNA targets of miR-608. We assessed the effects of miR-608 on the regulation of MIF in GSCs by transfecting pre-miR-608 or anti-miR-608. Real-time PCR results showed that the MIF mRNA expression was significantly decreased in the GSCs-U87 and GSCs-U251 cells transfected with the miR-608 mimic as compared to the respective NC, while miR-608 inhibition resulted in a significant increase in MIF
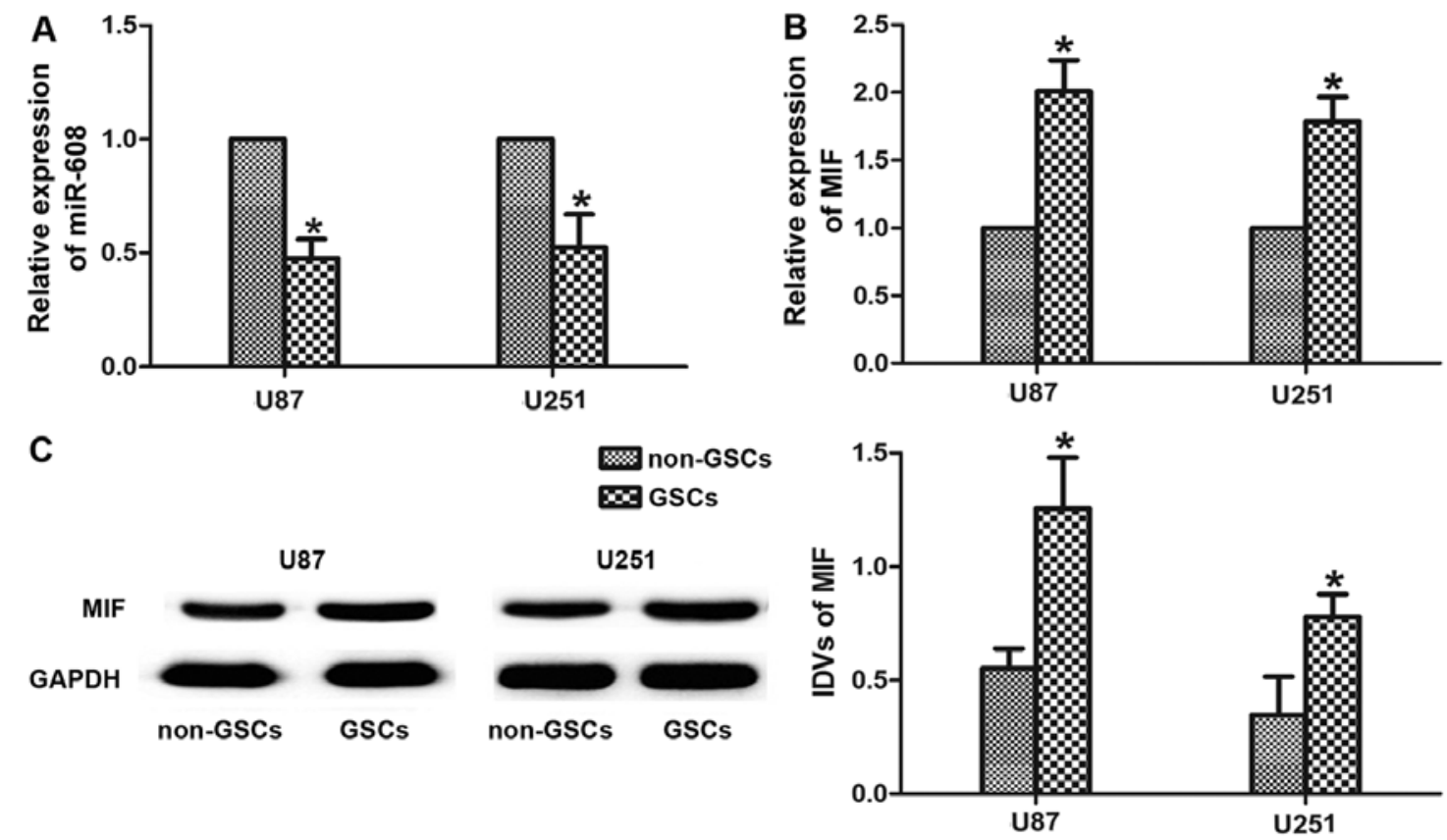

Figure 2. Expression levels of miR-608 and MIF in GSCs-U87 and GSCs-U251. (A) Quantitative real-time PCR analysis of the miR-608 expression in GSCs. The relative expression of miR-608 is shown using U6 as an endogenous control. (B) Quantitative real-time PCR analysis of the MIF gene in GSCs. The relative expression of MIF is shown using GAPDH as an endogenous control. (C) Western blot analysis of the protein level of MIF expression in GSCs. The IDVs of MIF are shown using GAPDH as an endogenous control. Data are expressed as the mean $\pm \mathrm{SD}\left(\mathrm{n}=5\right.$, each group). ${ }^{*}<0.05$ vs. the non-GSC group. 

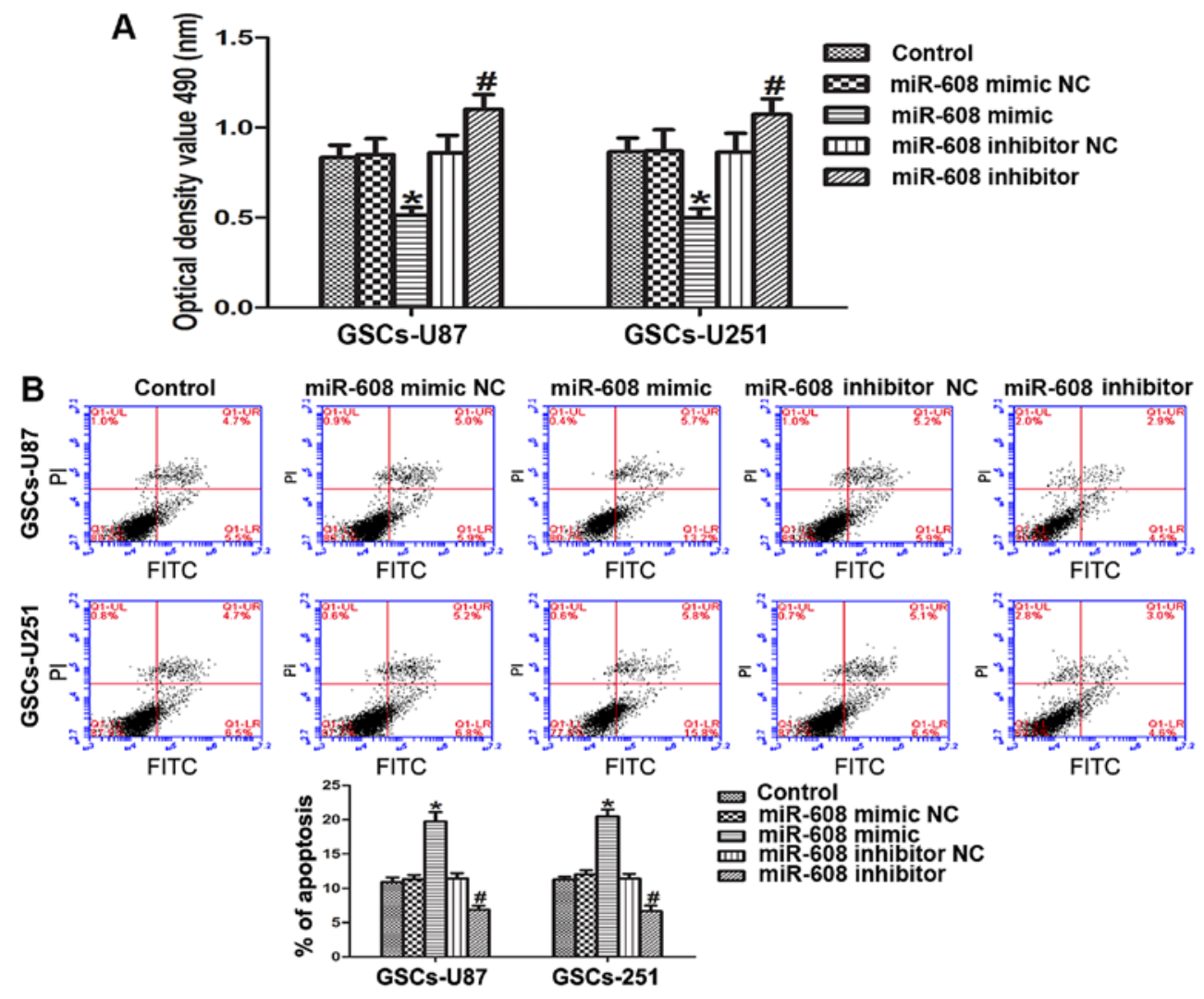

C Control miR-608 mimic NC miR-608 mimic miR-608 inhibitor NC miR-608 inhibitor
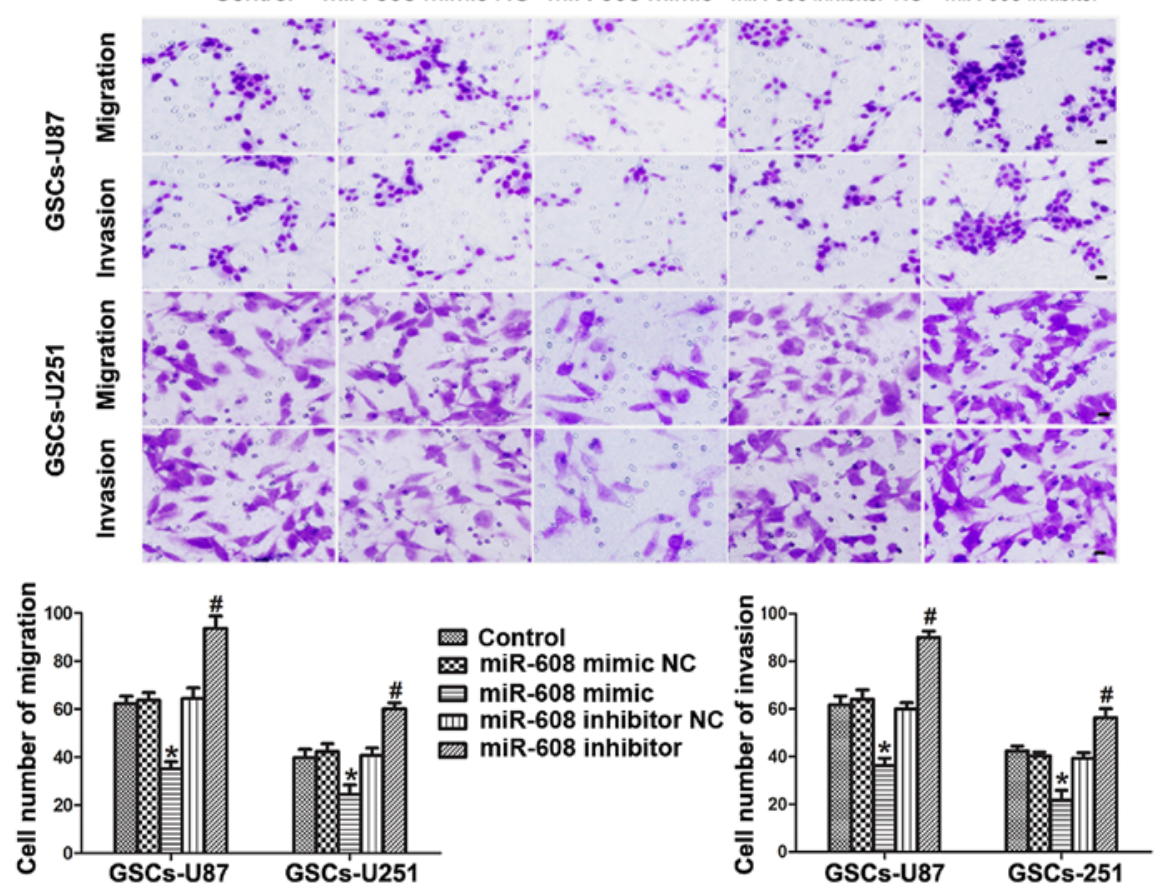

Figure 3. Effects of miR-608 on the malignant biological behavior of human GSCs isolated from the U87 and U251 cell lines. (A) The proliferative ability of GSCs transfected with the miR-608 mimic or inhibitor was examined by MTT assay. (B) Effects of miR-608 on the apoptosis of GSCs were evaluated by flow cytometric analysis. (C) Detection of cell migration and invasion abilities of the GSCs transfected with the miR-608 mimic or inhibitor. Representative graphs are shown. Data are expressed as the mean $\pm \mathrm{SD}\left(\mathrm{n}=5\right.$, each). ${ }^{*} \mathrm{P}<0.05$ vs. the miR-608 mimic NC group, ${ }^{\prime \prime} \mathrm{P}<0.05$ vs. the miR-608 inhibitor NC group. Scale bars, $20 \mu \mathrm{m}$.

expression in the GSCs (Fig. 4A). The effects of miR-608 overexpression or inhibition on the protein expression levels of MIF in GSCs were consistent with those of MIF mRNA (Fig. 4B).
MIF was predicted to have one miR-608 binding site in the 3'UTR using TargetScan 6.2. A dual-luciferase reporter system was used to determine whether MIF 3'UTR is a direct target of miR-608. miR-608 mimic and MIF 3'UTR-Wt 

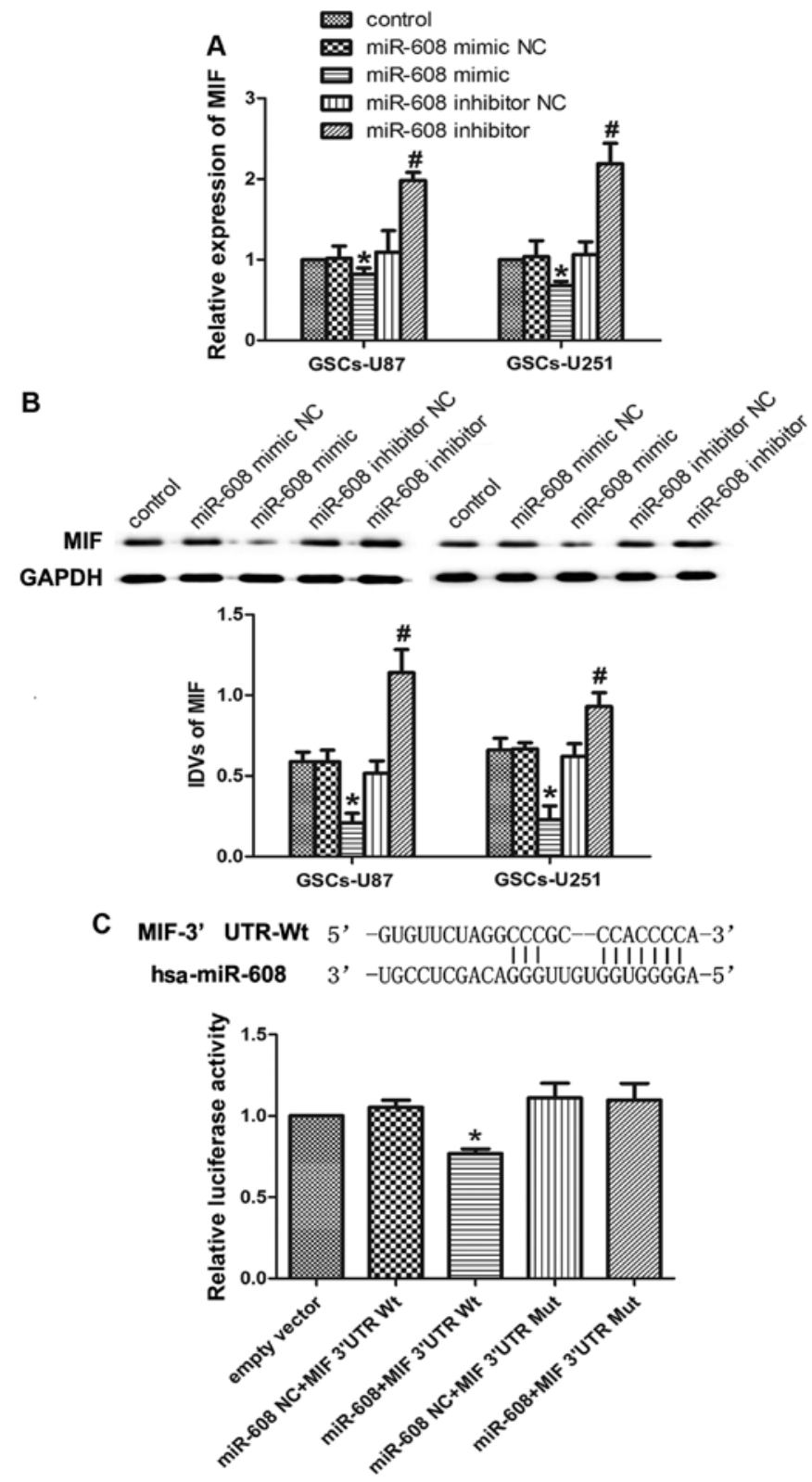

Figure 4. miR-608 negatively regulates MIF expression by targeting its 3'UTR. (A) Quantitative real-time PCR analysis of the MIF expression regulated by miR-608 in GSCs-U87 and GSCs-U251. (B) Western blot analysis of the MIF expression regulated by miR-608 in GSCs-U87 and GSCs-U251 The IDVs of MIF are shown using GAPDH as an endogenous control. ${ }^{*} \mathrm{P}<0.05$ vs. the miR-608 mimic $\mathrm{NC}$ group, ${ }^{\prime} \mathrm{P}<0.05$ vs. the miR-608 inhibitor NC group. (C) The predicted miR-608 binding sites in the 3'UTR region of MIF (MIF-3'UTR-Wt) and the designed mutant sequence (MIF-3'UTR-Mut) are presented. Luciferase reporter assay of HEK 293T cells transfected with MIF-3'UTR-Wt (or the MIF-3'UTR-Mut) and miRNA-608 mimic (or miRNA-608 NC) is shown. Data are expressed as the mean $\pm \mathrm{SD}(\mathrm{n}=5$, each group). ${ }^{*} \mathrm{P}<0.05$ vs. miR-608 mimic NC + MIF-3'UTR-Wt group.

reporter were co-transfected into HEK 293T cells and luciferase activity was measured. The relative luciferase activity in the cells co-transfected with the miR-608 mimic and MIF 3'UTR-Wt was significantly attenuated compared with that in miR-608 NC and MIF 3'UTR-Wt group, while co-transfection of the miR-608-NC and MIF 3'UTR-Mut did not change the luciferase activity (Fig. 4C). All these results suggest that MIF is a direct target of miR-608 with the specific binding site, and
miR-608 negatively regulates the expression of MIF gene and protein.

miR-608 overexpression inhibits the cell proliferation, migration and invasion, and promotes the apoptosis of GSCs by downregulating MIF. To explore whether miR-608 plays a tumor-suppressive role by regulating MIF, we assessed the ability of proliferation, migration, invasion and apoptosis after expression levels of miR-608 and MIF in the GSCs-U87 and GSCs-U251 cells were altered simultaneously. miR-608 overexpression combined with MIF inhibition significantly suppressed the proliferation of the GSCs, while the miR-608 inhibitor combined with MIF overexpression resulted in increased proliferation of GSCs in comparison with the control group. Moreover, the proliferation ability of GSCs with miR-608 overexpression combined with MIF inhibition was significantly attenuated compared with miR-608 overexpression combined with MIF overexpression. Similarly, miR-608 inhibition combined with MIF inhibition resulted in decreased proliferation compared with miR-608 inhibition combined with MIF overexpression (Fig. 5A). Furthermore, MTT assay data showed that miR- 608 overexpression combined with MIF inhibition led to a significant increase in the apoptosis rate, while miR-608 inhibition combined with MIF overexpression induced a significant reduction in apoptosis compared with the control in the GSCs-U87 and GSCs-U251. In addition, MIF inhibition combined with miR-608 overexpression or inhibition resulted in increased apoptosis compared with MIF overexpression combined with miR-608 overexpression or inhibition groups (Fig. 5B). The migration and invasion data showed a similar tendency with the MTT results. miR-608 overexpression combined with MIF inhibition attenuated the migration and invasion of the GSCs-U87 and GSCs-U251, and miR-608 inhibition combined with MIF overexpression increased the migration and invasion abilities of the GSCs compared with these abilities noted in the control. Furthermore, MIF inhibition combined with miR-608 overexpression or inhibition significantly decreased the migration and invasion abilities of the GSCs compared with these abilities in the MIF overexpression combined with miR-608 overexpression or inhibition groups (Fig. 5C). These results revealed that miR-608 plays a tumor-suppressive role by downregulating MIF.

miR-608 overexpression inhibits the PI3K/AKT and JNK pathways by downregulating MIF in GSCs. To determine whether the PI3K/AKT and JNK pathways are involved in the tumor-suppressive effects of miR-608 in GSCs, we detected the protein expression levels of p-PI3K, p-AKT and p-JNK after the expression levels of miR-608 and MIF in the GSCs-U87 or GSCs-U251 cells were altered at the same time. As shown in Fig. 6A, the expression level of the p-PI3K protein was significantly lower in the miR-608 overexpression combined with MIF inhibition group, and highest in the miR-608 inhibition combined with MIF overexpression group compared with the control group. Furthermore, MIF inhibition combined with miR-608 overexpression or inhibition significantly inhibited the activation of p-PI3K compared with the MIF overexpression combined with miR-608 overexpression or inhibition groups. The expression levels of phosphorylated AKT and JNK were consistent with the results of p-PI3K 


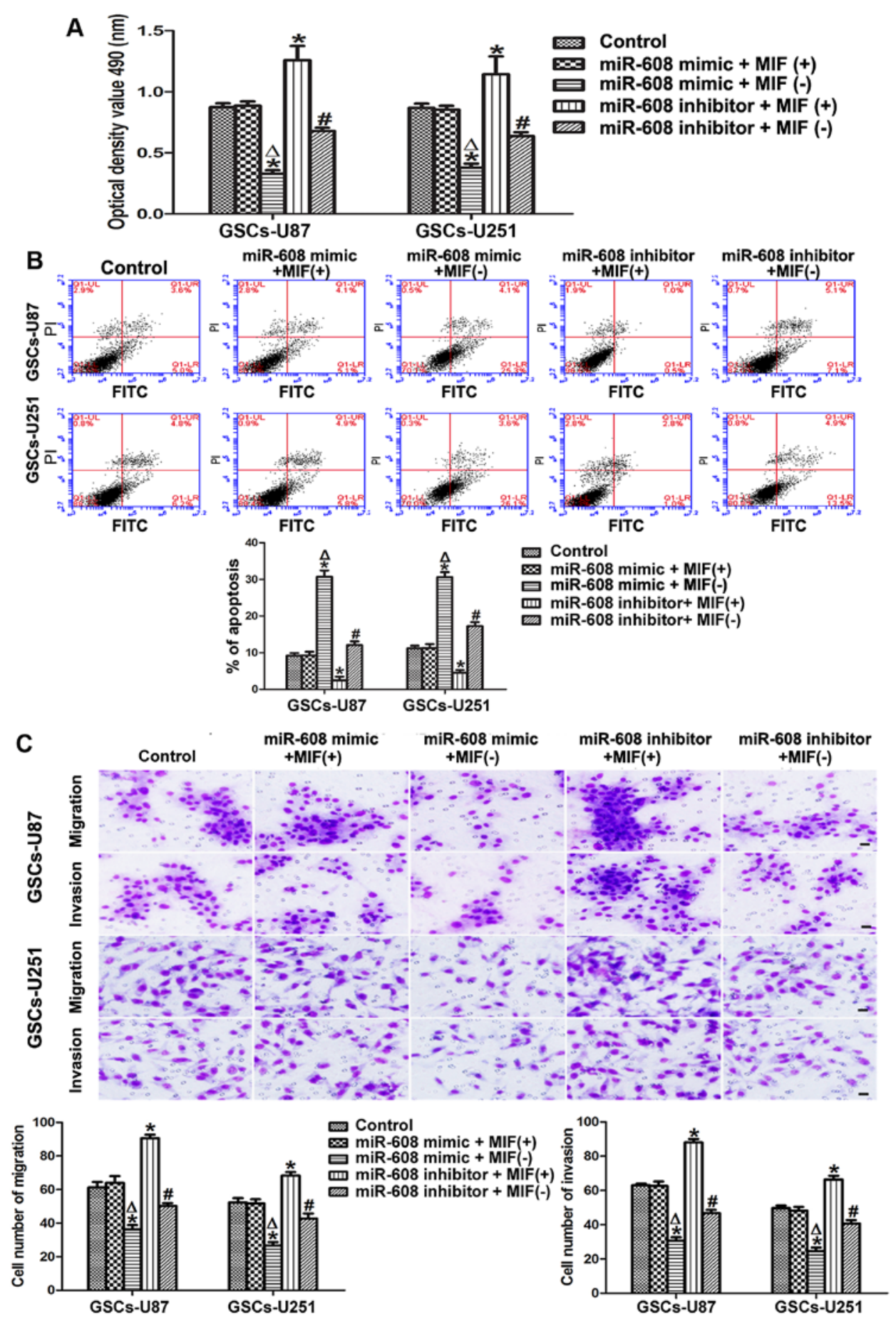

Figure 5. miR-608 overexpression inhibits the cell proliferation, migration, invasion and promotes the apoptosis of GSCs-U87 and GSCs-U251 by downregulating MIF. (A) MTT assay to test the proliferative effect of miR-608 on GSCs-U87 and GSCs-U251 by downregulating MIF. (B) Analysis of apoptosis of GSCs-U87 and GSCs-U251 regulated by miR-608 and MIF. (C) The cell number of migratory and invasive GSCs-U87 and GSCs-U251 as regulated by miR-608 and MIF. Representative images and corressponding statistical data are presented. Data are presented as the mean $\pm \mathrm{SD}\left(\mathrm{n}=5\right.$, each group). ${ }^{*} \mathrm{P}<0.05$ vs. the control group, ${ }^{\Delta} \mathrm{P}<0.05$ vs. the miR-608 mimic $+\mathrm{MIF}(+)$ group, ${ }^{*} \mathrm{P}<0.05$ vs. the miR-608 inhibitor+ $\mathrm{MIF}(+)$ group. Scale bars, $20 \mu \mathrm{m}$.

in the GSCs-U87 and GSCs-U251 cells as determined by western blotting (Fig. 6B and $\mathrm{C}$ ). These results suggest that the PI3K/AKT and JNK pathways may be involved in the tumor-suppressive effects of miR-608 in GSCs.

\section{Discussion}

The present study demonstrated that miR-608 exhibited a low expression level in glioblastoma tissues and GSCs which were isolated from the U87 and U251 cell lines, and the overexpression of miR-608 inhibited the proliferation, migration and invasion and induced the apoptosis of the GSCs. Moreover, the results of the dual-luciferase reporter assay and bioinformatic databases showed that MIF is a direct target gene of miR-608, and miR-608 downregulated the expression level of MIF in the GSCs. Subsequently, high expression levels of MIF mRNA and protein were verified in GSCs. Moreover, we found that miR-608 plays a tumor-suppressive role by targeting MIF, and 

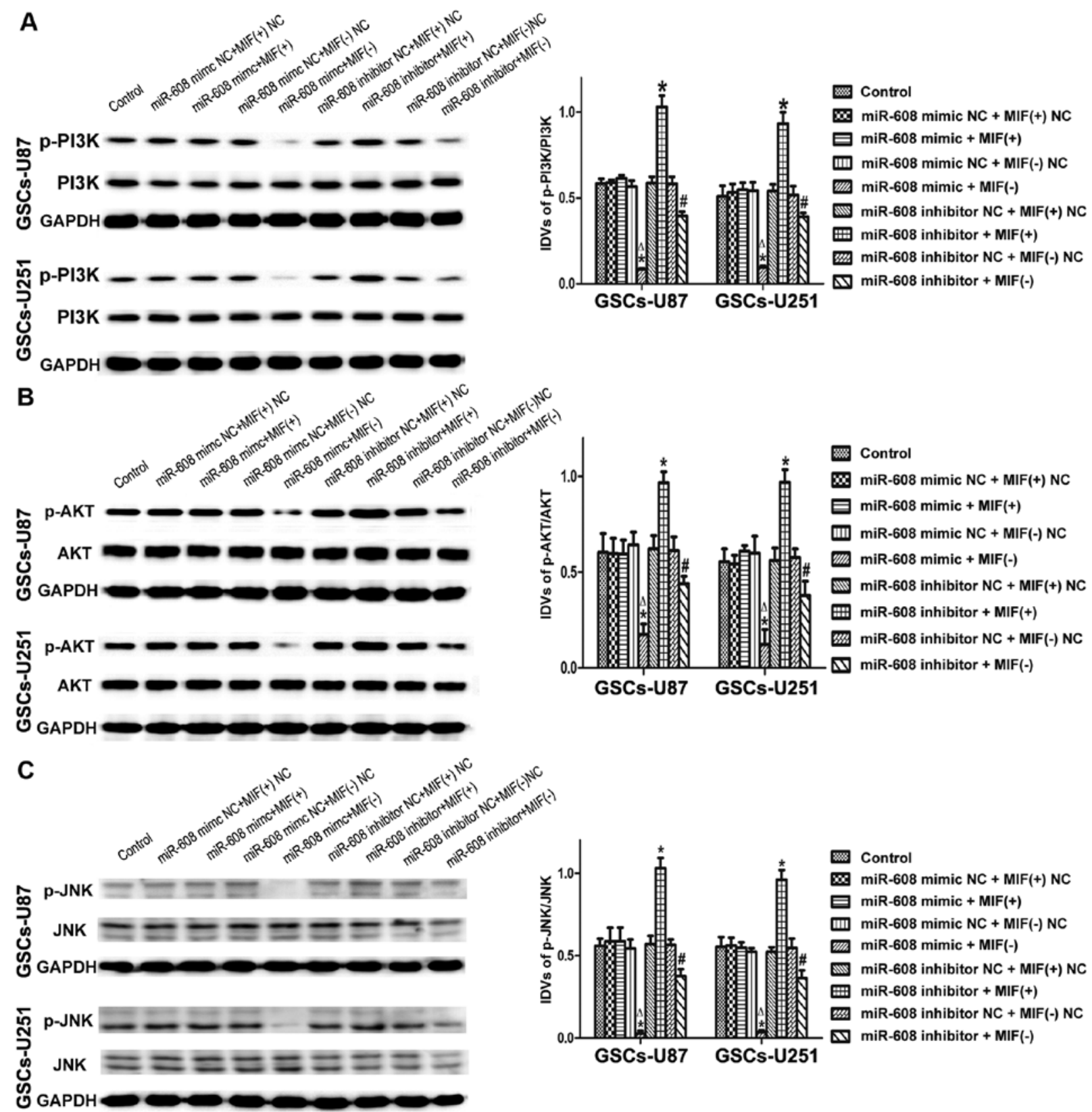

Figure 6. miR-608 overexpression inhibits the activation of PI3K/AKT and JNK pathways in GSCs-U87 and GSCs-U251 by downregulating MIF. (A) Western blot analysis of the p-PI3K/PI3K expression regulated by miR-608 and MIF in GSCs-U87 and GSCs-U251. (B) Western blot analysis of the p-AKT/AKT expression regulated by miR-608 and MIF in GSCs-U87 and GSCs-U251. (C) Western blot analysis of the p-JNK/JNK expression regulated by miR-608 and MIF in GSCs-U87 and GSCs-U251. Data are expressed as the mean $\pm \mathrm{SD}\left(\mathrm{n}=5\right.$, each group). ${ }^{*} \mathrm{P}<0.05$ vs. the control group, ${ }^{\Delta} \mathrm{P}<0.05$ vs. the miR-608 mimic $+\mathrm{MIF}(+)$ group, ${ }^{\#} \mathrm{P}<0.05$ vs. the miR-608 inhibitor $+\mathrm{MIF}(+)$ group.

the PI3K/AKT and JNK signaling pathways may be involved in this process.

Glioma is a type of prevalent central nervous system cancer which seriously threatens human health. In particular, GBM is a fatal malignancy characterized by a highly invasive nature, devastating recurrence and short survival period $(18,19)$. To date, the current treatment for glioma can not yield the desired long-term survival of patients due to rapid tumor growth and limitation of the blood-brain barrier. Moreover, the high heterogeneity of glioma in regards to growth, invasive ability, therapeutic effect, prognosis and so on further exacerbates the difficulty and complexity of treatment. Moreover, high-level individualized treatment for glioma has not yet been real- ized. Recently, investigators found that there exists cancer stem-like cells in glioma, to a large extent, contributing to the tumorigenicity and chemoresistance. These GSCs have self-renewal capacity and multipotency of differentiation as well as the ability to initiate tumor formation (20-22). Elucidation of the detailed molecular mechanisms and related molecules that regulate GSCs may offer a promising choice for targeted therapeutic methods. In this study, the qRT-PCR data showed that the expression of miR-608 was significantly lower in GSCs compared with non-GSCs. Yet, miR-608 is relatively unexplored and little is known about its function. In recent years, some studies have reported the association between a single-nucleotide polymorphisms 
(SNPs) in hsa-mir-608 and several cancers including nasopharyngeal carcinoma, colorectal cancer, breast cancer, and renal cell carcinoma (23-26). In addition, it has been reported that miR-608 is downregulated in chordoma cells, acting as a tumor suppressor (7). Our subsequent experiments indicated that overexpression of miR-608 inhibits the proliferation, migration and invasion and promotes the apoptosis of GSCs, while the knockout of miR-608 led to reverse effects. The results suggest that downregulation of miR-608 in GSCs is implicated in the malignant development of glioma.

Zhang et al (7) revealed that EGFR and Bcl-xL are target genes of miR-608 in chordoma cells, and miR-608 may regulate chordoma proliferation and invasion by targeting EGFR and Bcl-xL. To explore the target gene of miR-608 in GSCs, we predicted that the 3'UTR of human MIF contained binding sites of base positions with miR-608 using bioinformatic tools. Furthermore, we found that the expression levels of MIF mRNA and protein were significantly enhanced in GSCs in comparison to non-GSCs. Actually, it has been proven that MIF is highly expressed in human glioma tissues and in a series of glioma cell lines. Moreover, glioma cell-derived MIF plays a crucial role in tumor progression and angiogenesis as well as the immune escape of malignant gliomas (8). MIF, as a cytokine, is overexpressed in many tumors, such as esophageal, colon, prostate cancers and GBM. It has already been regarded as a promising target molecule for the treatment of various types of tumors. One study showed that ISO, an inhibitor of MIF, reduced the cell growth rate and invasive ability of GBM (27). Our new finding was that high expression of MIF was observed in GSCs-U87 and GSCs-U251. The luciferase assay further confirmed that luciferase activities of 293T cells co-transfected with miR-608 mimic and MIF 3'UTR-Wt were significantly inhibited, suggesting that there exist binding sites between miR-608 and MIF which coincided with the predicted results of the bioinformatic software. Additionally, qRT-PCR and western blot results also verified that GSCs transfected with the miR-608 mimic had attenuated expression of MIF mRNA and protein, while MIF presented a high expression in GSCs transfected with the miR-608 inhibitor. Taken together, these results reinforce the notion that MIF is involved in the molecular mechanism of miR-608 in the regulation of the malignancy of GSCs which highly elucidates the role of MIF in glioma.

To demonstrate whether MIF also plays an oncogenic role in GSCs, and whether miR-608 could influence the tumor progression of GSCs via regulating MIF, we constructed a GSC model co-transfected with miR-608 and MIF. A series of experiments proved that MIF overexpression combined with miR-608 inhibition significantly decreased the apoptosis and promoted the proliferation, migration and invasion of GSCs. By contrast, miR-608 overexpression combined with MIF inhibition led to opposite results. Therefore, we conclude that GSC-derived MIF, as a potential target molecule of miR-608 plays an indispensible role in the malignant progression of GSCs.

Baron et al (27) revealed that MIF may bind to the CD74/ CD44 receptor complex and promote migration, invasion, and proliferation by activating the ERK1/2 MAPK cascade in GBM tumor cell lines. Inhibition of PI3K signaling has been thought to be a potential adjuvant strategy for glioma therapy $(10,28)$.
Our laboratory team reported that endothelial-monocyte activating polypeptide-II (EMAP-II), an anticancer agent, may inhibit the activation of PI3K/Akt pathway in GSCs (29). In this study, we found that the expression levels of $\mathrm{p}-\mathrm{PI} 3 \mathrm{~K}$ and p-AKT protein in the co-transfected GSCs with miR-608 overexpression combined with MIF inhibition were significantly lower than those in the control group, while GSCs with miR-608 inhibition combined with MIF overexpression significantly activated PI3K/AKT signaling, suggesting that the PI3K/Akt pathway was mediated by the effects of miR-608 on GSCs via regulation of MIF. Together with the PI3K/AKT pathway, the role of the JNK pathway was also investegated in this study. Among the three major MAP kinase families including extracellular signal-regulated kinases (ERK), JNK and p38, JNK is thought to be closely associated with the maintenance of GSCs. And targeting JNK may be a viable, clinically treatment for depletion of GSCs (12). Our data showed that MIF overexpression and miR-608 knockdown could elevate the expression of p-JNK protein in GSCs, which heightened the findings that the JNK pathway may play a cancer-promoting role in glioma.

In conclusion, we reported for the first time that overexpression of miR-608 inhibited the cell proliferation, migration, invasion, and promoted the apoptosis of GSCs isolated from the U87 and U251 cell lines by targeting MIF, and the JNK and PI3K/AKT signaling pathways may be involved in this biological process. The results indicated that miR-608 could be used as a potential target miRNA for the treatment of human glioblastoma.

\section{Acknowledgements}

The present study was funded by the following grants: contract grant sponsor Natural Science Foundation, China, contract grant no. 81201800; contract grant sponsor Shenyang Science and Technology Plan Projects, China, contract grant no. F12-277-1-68; contract grant sponsor Natural Science Foundation of Liaoning Province, China, contract grant no. 2015020750.

\section{References}

1. Galli R, Binda E, Orfanelli U, Cipelletti B, Gritti A, De Vitis S, Fiocco R, Foroni C, Dimeco F and Vescovi A: Isolation and characterization of tumorigenic, stem-like neural precursors from human glioblastoma. Cancer Res 64: 7011-7021, 2004.

2. Lathia JD, Mack SC, Mulkearns-Hubert EE, Valentim CL and Rich JN: Cancer stem cells in glioblastoma. Genes Dev 29: 1203-1217, 2015.

3. Bao S, Wu Q, McLendon RE, Hao Y, Shi Q, Hjelmeland AB, Dewhirst MW, Bigner DD and Rich JN: Glioma stem cells promote radioresistance by preferential activation of the DNA damage response. Nature 444: 756-760, 2006.

4. Chen J, Li Y, Yu TS, McKay RM, Burns DK, Kernie SG and Parada LF: A restricted cell population propagates glioblastoma growth after chemotherapy. Nature 488: 522-526, 2012.

5. Seymour T, Nowak A and Kakulas F: Targeting aggressive cancer stem cells in glioblastoma. Front Oncol 5: 159, 2015.

6. Liu S, Yin F, Zhang J, Wicha MS, Chang AE, Fan W, Chen L, Fan M and Li Q: Regulatory roles of miRNA in the human neural stem cell transformation to glioma stem cells. J Cell Biochem 115: 1368-1380, 2014.

7. Zhang Y, Schiff D, Park D and Abounader R: MicroRNA-608 and microRNA-34a regulate chordoma malignancy by targeting EGFR, Bcl-xL and MET. PLoS One 9: e91546, 2014. 
8. Mittelbronn M, Platten M, Zeiner P, Dombrowski Y, Frank B, Zachskorn C, Harter PN, Weller $M$ and Wischhusen $J$ : Macrophage migration inhibitory factor (MIF) expression in human malignant gliomas contributes to immune escape and tumour progression. Acta Neuropathol 122: 353-365, 2011.

9. Wang XB, Tian XY, Li Y, Li B and Li Z: Elevated expression of macrophage migration inhibitory factor correlates with tumor recurrence and poor prognosis of patients with gliomas. J Neurooncol 106: 43-51, 2012.

10. Ströbele S, Schneider M, Schneele L, Siegelin MD, Nonnenmacher L, Zhou S, Karpel-Massler G, Westhoff MA, Halatsch ME and Debatin KM: A potential role for the inhibition of PI3K signaling in glioblastoma therapy. PLoS One 10 e0131670, 2015.

11. Liu J and Lin A: Role of JNK activation in apoptosis: A double-edged sword. Cell Res 15: 36-42, 2005.

12. Matsuda K, Sato A, Okada M, Shibuya K, Seino S, Suzuki K, Watanabe E, Narita Y, Shibui S, Kayama T, et al: Targeting JNK for therapeutic depletion of stem-like glioblastoma cells. Sci Rep 2: 516, 2012.

13. Alapati K, Kesanakurti D, Rao JS and Dasari VR: uPAR and cathepsin B-mediated compartmentalization of JNK regulates the migration of glioma-initiating cells. Stem Cell Res (Amst) 12: 716-729, 2014.

14. Lue H, Dewor M, Leng L, Bucala R and Bernhagen J: Activation of the JNK signalling pathway by macrophage migration inhibitory factor (MIF) and dependence on CXCR4 and CD74. Cell Signal 23: 135-144, 2011.

15. Yeh TM, Liu SH, Lin KC, Kuo C, Kuo SY, Huang TY, Yen YR, Wen RK, Chen LC and Fu TF: Dengue virus enhances thrombomodulin and ICAM-1 expression through the macrophage migration inhibitory factor induction of the MAPK and PI3K signaling pathways. PLoS One 8: e55018, 2013.

16. Zhang FL, Wang P, Liu YH, Liu LB, Liu XB, Li Z and Xue YX: Topoisomerase I inhibitors, shikonin and topotecan, inhibit growth and induce apoptosis of glioma cells and glioma stem cells. PLoS One 8: e81815, 2013.

17. Yao Y, Xue Y, Ma J, Shang C, Wang P, Liu L, Liu W, Li Z, Qu S, $\mathrm{Li} Z$, et al: miR-330-mediated regulation of SH3GL2 expression enhances malignant behaviors of glioblastoma stem cells by activating ERK and PI3K/AKT signaling pathways. PLoS One 9: e95060, 2014.

18. Stupp R, Mason WP, van den Bent MJ, Weller M, Fisher B, Taphoorn MJ, Belanger K, Brandes AA, Marosi C, Bogdahn U, et al; European Organisation for Research and Treatment of Cancer Brain Tumor and Radiotherapy Groups; National Cancer Institute of Canada Clinical Trials Group: Radiotherapy plus concomitant and adjuvant temozolomide for glioblastoma. N Engl J Med 352: 987-996, 2005.
19. Khasraw $M$ and Lassman AB: Advances in the treatment of malignant gliomas. Curr Oncol Rep 12: 26-33, 2010.

20. Reya T, Morrison SJ, Clarke MF and Weissman IL: Stem cells, cancer, and cancer stem cells. Nature 414: 105-111, 2001.

21. Clevers H: The cancer stem cell: Premises, promises and challenges. Nat Med 17: 313-319, 2011.

22. Malik B and Nie D: Cancer stem cells and resistance to chemo and radio therapy. Front Biosci (Elite Ed) 4: 2142-2149, 2012

23. Lin J, Horikawa Y, Tamboli P, Clague J, Wood CG and Wu X Genetic variations in microRNA-related genes are associated with survival and recurrence in patients with renal cell carcinoma. Carcinogenesis 31: 1805-1812, 2010.

24. Huang AJ, Yu KD, Li J, Fan L and Shao ZM: Polymorphism rs4919510:C $>\mathrm{G}$ in mature sequence of human microRNA-608 contributes to the risk of HER2-positive breast cancer but not other subtypes. PLoS One 7: e35252, 2012.

25. Ryan BM, McClary AC, Valeri N, Robinson D, Paone A, Bowman ED, Robles AI, Croce C and Harris CC: rs4919510 in hsa-mir-608 is associated with outcome but not risk of colorectal cancer. PLoS One 7: e36306, 2012.

26. Qiu F, Yang L, Zhang L, Yang X, Yang R, Fang W, Wu D, Chen J, Xie C, Huang D, et al: Polymorphism in mature microRNA-608 sequence is associated with an increased risk of nasopharyngeal carcinoma. Gene 565: 180-186, 2015.

27. Baron N, Deuster O, Noelker C, Stüer C, Strik H, Schaller C, Dodel R, Meyer B and Bacher M: Role of macrophage migration inhibitory factor in primary glioblastoma multiforme cells. J Neurosci Res 89: 711-717, 2011.

28. Shi L, Fei X, Wang Z and You Y: PI3K inhibitor combined with miR-125b inhibitor sensitize TMZ-induced anti-glioma stem cancer effects through inactivation of $\mathrm{Wnt} / \beta$-catenin signaling pathway. In Vitro Cell Dev Biol Anim 51: 1047-1055, 2015.

29. Liu J, Liu L, Xue Y, Meng F, Li S, Wang P and Liu Y: Anti-neoplastic activity of low-dose endothelial-monocyte activating polypeptide-II results from defective autophagy and G2/M arrest mediated by PI3K/Akt/FoxO1 axis in human glioblastoma stem cells. Biochem Pharmacol 89: 477-489, 2014. 